\title{
ASYMPTOTIC BEHAVIOUR OF EIGEN-VALUES OF CERTAIN INTEGRAL EQUATIONS
}

\author{
by J. B. READE \\ (Received 15th November 1977)
}

\section{Introduction}

The integral equations we consider are

$$
\int_{-1}^{1} \log |x-t| f(t) d t=\lambda f(x)
$$

and

$$
\int_{-1}^{1} \frac{1}{|x-t|^{\alpha}} f(t) d t=\lambda f(x)
$$

where $0<\alpha<1$. The asymptotic behaviour of the eigen-values of the latter equation is already known (see (1) and (4)). The former equation has been studied by many authors but as yet no explicit statement seems to have been made about the behaviour of its eigen-values.

We shall show that the eigen-values of the first equation are all negative and tend to zero with order $1 / n$, and that the eigen-values of the second equation are all positive and tend to zero with order $1 / n^{1-\alpha}$. These results agree with those of (1), (4) though our methods are quite different.

We give the full details for the equation

$$
\int_{-1}^{1} \log |x-t| f(t) d t=\lambda f(x)
$$

and indicate briefly how the arguments can be adapted to cover the other equation.

2.

We need the following expansion of the kernel $\log |x-t|$ in Chebyshev polynomials.

Lemma 1. For all $-1<x \neq t<1$,

$$
\log |x-t|=-\log 2-\sum_{n=1}^{\infty} \frac{2}{n} T_{n}(x) T_{n}(t)
$$

where $T_{n}(\cos \theta)=\cos n \theta$. 
Proof. Writing $x=\cos \theta, t=\cos \phi(0<\theta \neq \phi<\pi)$ we have

$$
\begin{aligned}
\sum_{n=1}^{\infty} \frac{2}{n} T_{n}(x) T_{n}(t) & =\sum_{n=1}^{\infty} \frac{2}{n} \cos n \theta \cos n \phi \\
& =\sum_{n=1}^{\infty} \frac{1}{n}\{\cos n(\theta+\phi)+\cos n(\theta-\phi)\} \\
& =\operatorname{Re}\left\{-\log \left(1-\mathrm{e}^{i(\theta+\phi)}\right)\left(1-\mathrm{e}^{i(\theta-\phi)}\right)\right\} \\
& =-\log \left|\left(1-e^{i(\theta+\phi)}\right)\left(1-e^{i(\theta-\phi)}\right)\right| \\
& =-\log 4 \sin \frac{1}{2}(\theta+\phi) \sin \frac{1}{2}|\theta-\phi| \\
& =-\log 2|\cos \theta-\cos \phi| \\
& =-\log 2|x-t|
\end{aligned}
$$

from which the lemma follows.

\section{Corollary.}

$$
\begin{aligned}
\int_{-1}^{1} \log |x-t| T_{n}(t)\left(1-t^{2}\right)^{-1 / 2} d t & =-\pi \log 2 \quad(n=0) \\
& =-\frac{\pi}{n} T_{n}(x) \quad(n \geqslant 1)
\end{aligned}
$$

3.

The above corollary does not give any direct information about the eigen-values of the equation

$$
\int_{-1}^{1} \log |x-t| f(t) d t=\lambda f(x)
$$

but it does say something about the equation

$$
\int_{-1}^{1}\left(1-x^{2}\right)^{-1 / 4} \log |x-t|\left(1-t^{2}\right)^{-1 / 4} f(t) d t=\lambda f(x)
$$

namely that its eigen-functions are $\left\{\left(1-x^{2}\right)^{-1 / 4} T_{n}(x)\right\}_{n \geqslant 0}$ with eigen-values $-\pi \log 2$ $(n=0)$ and $-\pi / n(n \geqslant 1)$. We relate these eigen-values to the eigen-values of

$$
\int_{-1}^{1} \log |x-t| f(t) d t=\lambda f(x)
$$

by means of the following general lemma about operators on Hilbert space.

Lemma 2. If $T$ is a positive compact operator on a Hilbert space $H$, and if $M$ is a self-adjoint operator on $H$ with $\|M\| \leqslant 1$, then $M T M$ is positive and if the eigen-values of $T, M T M$ are respectively

$$
\begin{aligned}
& \lambda_{1} \geqslant \lambda_{2} \geqslant \cdots \\
& \mu_{1} \geqslant \mu_{2} \geqslant \cdots
\end{aligned}
$$

then $\mu_{n} \leqslant \lambda_{n}$ for all $n$. 
Proof. For all $f \in H$ we have

$$
\begin{aligned}
\langle M T M f, f\rangle & =\langle T M f, M f\rangle \\
& \geqslant 0
\end{aligned}
$$

therefore $M T M \geqslant 0$.

To prove the assertion about the eigen-values we use the Weyl-Courant minimax principle in the form

$$
\lambda_{n}=\inf _{R}\|T-R\|
$$

where the infimum is taken over all operators $R$ of rank $\leqslant n-1$. (See e.g. (2) page 132.)

We have

$$
\begin{aligned}
\mu_{n} & =\inf _{R}\|M T M-R\| \\
& \leqslant \inf _{R}\|M T M-M R M\|,
\end{aligned}
$$

since $M R M$ also has rank $\leqslant n-1$,

$$
\begin{aligned}
& =\inf _{R}\|M(T-R) M\| \\
& \leqslant \inf _{R}\|M\|\|T-R\|\|M\| \\
& \leqslant \inf _{R}\|T-R\| \\
& =\lambda_{n} .
\end{aligned}
$$

If we take

$$
\begin{gathered}
T f(x)=-\int_{-1}^{1}\left(1-x^{2}\right)^{-1 / 4} \log |x-t|\left(1-t^{2}\right)^{-1 / 4} f(t) d t \\
M f(x)=\left(1-x^{2}\right)^{1 / 4} f(x)
\end{gathered}
$$

then we have

$$
\operatorname{MTM} f(x)=-\int_{-1}^{1} \log |x-t| f(t) d t
$$

so we deduce from Lemma 2 that all the eigen-values of

$$
\int_{-1}^{1} \log |x-t| f(t) d t=\lambda f(x)
$$

are negative and if we denote them by

$$
\lambda_{0} \leqslant \lambda_{1} \leqslant \lambda_{2} \leqslant \cdots \quad(\leqslant 0)
$$


then

$$
\begin{aligned}
& \left|\lambda_{0}\right| \leqslant \pi \log 2, \\
& \left|\lambda_{n}\right| \leqslant \pi / n \quad(n \geqslant 1) .
\end{aligned}
$$

4.

We obtain lower bounds for $\left|\lambda_{n}\right|$ by expanding $\log |x-t|$ in Chebyshev polynomials of the second kind. We have from Lemma 1

$$
\begin{aligned}
\log |x-t|= & -\log 2-\sum_{n=1}^{\infty} \frac{2}{n} T_{n}(x) T_{n}(t) \\
= & -\log 2-\frac{1}{2} U_{1}(x) U_{1}(t) \\
& -\sum_{n=2}^{\infty} \frac{1}{2 n}\left(U_{n}(x)-U_{n-2}(x)\right)\left(U_{n}(t)-U_{n-2}(t)\right),
\end{aligned}
$$

where $U_{n}(\cos \theta)=\sin (n+1) \theta / \sin \theta$,

$$
\begin{aligned}
= & -\log 2-\frac{1}{4}-\frac{1}{2} \sum_{n=1}^{\infty}\left(\frac{1}{n}+\frac{1}{n+2}\right) U_{n}(x) U_{n}(t) \\
& +\frac{1}{2} \sum_{n=0}^{\infty} \frac{1}{n+2}\left\{U_{n}(x) U_{n+2}(t)+U_{n+2}(x) U_{n}(t)\right\},
\end{aligned}
$$

which gives

$$
\begin{aligned}
\int_{-1}^{1} \log |x-t|\left(1-t^{2}\right)^{1 / 2} U_{n}(t) d t & =-\frac{\pi}{2} \log 2-\frac{\pi}{8}+\frac{\pi}{8} U_{2}(x) \quad(n=0) \\
& =-\frac{\pi}{4}\left(1+\frac{1}{3}\right) U_{1}(x)+\frac{\pi}{12} U_{3}(x) \quad(n=1) \\
& =\frac{\pi}{4 n} U_{n-2}(x)-\frac{\pi}{4}\left(\frac{1}{n}+\frac{1}{n+2}\right) U_{n}(x) \\
& +\frac{\pi}{4(n+2)} U_{n+2}(x) \quad(n \geqslant 2) .
\end{aligned}
$$

It follows that the operator

$$
U f(x)=-\int_{-1}^{1}\left(1-x^{2}\right)^{1 / 4} \log |x-t|\left(1-t^{2}\right)^{1 / 4} f(t) d t
$$

has matrix

$$
\frac{\pi}{4}\left(\begin{array}{cccc}
2 \log 2+\frac{1}{2} & 0- & --\frac{1}{2} & - \\
0 & 1+\frac{1}{3} & 0 & -\frac{1}{3} \cdot \\
-\frac{1}{2} & 0 & \frac{1}{2}+\frac{1}{4} \cdot & \\
& -\frac{1}{3} \cdot & &
\end{array}\right)
$$


with respect to the orthonormal basis $\left\{(2 / \pi)^{1 / 2}\left(1-x^{2}\right)^{1 / 4} U_{n}(x)\right\}_{n \geqslant 0}$ of the Hilbert space $L^{2}[-1,1]$. Now in the notation of Section 3 we have $U=M T M$ and so by Lemma 2 , $U$ is positive and, if we denote its eigen-values by

$$
\mu_{0} \geqslant \mu_{1} \geqslant \mu_{2} \geqslant \cdots,
$$

then $\left|\lambda_{n}\right| \geqslant \mu_{n}$ for all $n$.

5.

We estimate $\mu_{n}$ by truncating the matrix for $U$ and comparing the truncates with tridiagonal matrices with constant diagonals whose eigen-values can be found explicitly.

If $f \in L^{2}[-1,1]$ has Fourier-Chebyshev coefficients of the second kind $\left(c_{n}\right)$ then

$$
\begin{aligned}
\langle U f, f\rangle= & \frac{\pi}{4}\left\{\left(2 \log 2+\frac{1}{2}\right) c_{0}^{2}+\sum_{n=1}^{\infty}\left(\frac{1}{n}+\frac{1}{n+2}\right) c_{n}^{2}-\sum_{n=0}^{\infty} \frac{2}{n+2} c_{n} c_{n+2}\right\} \\
= & \frac{\pi}{4}\left\{\left(2 \log 2+\frac{1}{2}\right) c_{0}^{2}+\frac{1}{2}\left(c_{0}-c_{2}\right)^{2}+\cdots+\frac{1}{2 n}\left(c_{2 n-2}-c_{2 n}\right)^{2}+\cdots\right. \\
& \left.+c_{1}^{2}+\frac{1}{3}\left(c_{1}-c_{3}\right)^{2}+\cdots+\frac{1}{2 n-1}\left(c_{2 n-3}-c_{2 n-1}\right)^{2}+\cdots\right\} \\
\geqslant & \frac{\pi}{4}\left\{\left(2 \log 2+\frac{1}{2}\right) c_{0}^{2}+\frac{1}{2}\left(c_{0}-c_{2}\right)^{2}+\cdots+\frac{1}{2 N}\left(c_{2 N-2}-c_{2 N}\right)^{2}\right. \\
& \left.+c_{1}^{2}+\frac{1}{3}\left(c_{1}-c_{3}\right)^{2}+\cdots+\frac{1}{2 N-1}\left(c_{2 N-3}-c_{2 N-1}\right)^{2}\right\} \\
\geqslant & \frac{\pi}{8 N}\left\{c_{0}^{2}+\left(c_{0}-c_{2}\right)^{2}+\cdots+\left(c_{2 N-2}-c_{2 N}\right)^{2}\right. \\
& \left.+c_{1}^{2}+\left(c_{1}-c_{3}\right)^{2}+\cdots+\left(c_{2 N-3}-c_{2 N-1}\right)^{2}\right\} \\
= & \frac{\pi}{8 N}\langle V f, f\rangle
\end{aligned}
$$

$V$ being the operator with matrix $\left(\begin{array}{l|l}A & 0 \\ \hline 0 & 0\end{array}\right)$ where

$$
A=\left(\begin{array}{rrrrrrr}
2 & 0 & -1 & & & & \\
0 & 2 & 0 & -1 & & \\
-1 & 0 & 2 & & \ddots & & \\
& -1 & & \ddots & & -1 & \\
& & \ddots & & 2 & 0 & -1 \\
& & -1 & 0 & 1 & 0 \\
& & & -1 & 0 & 1
\end{array}\right)
$$

of order $2 N+1$. If we denote the eigen-values of $A$ by

$$
\nu_{0} \geqslant \nu_{1} \geqslant \cdots \geqslant \nu_{2 N}
$$


then we have

for all $0 \leqslant n \leqslant 2 N$.

$$
\mu_{n} \geqslant(\pi /(8 N)) \nu_{n}
$$

Lemma 3. The eigen-values of the $N \times N$ matrix

are $\left\{4 \cos ^{2} \frac{\frac{1}{2} n \pi}{N+1}\right\}_{n=1,2, \ldots, N}$

$$
B_{N}=\left(\begin{array}{rrrrrr}
2 & -1 & & & & \\
-1 & 2 & -1 & & \\
& -1 & 2 & \ddots & \\
& & \ddots & \ddots & -1 & \\
& & & -1 & 2 & -1 \\
& & & & -1 & 2
\end{array}\right)
$$

Proof. The characteristic polynomial $p_{N}(\lambda)$ of $B_{N}$ satisfies the recurrence relation

$$
p_{N+2}(\lambda)=(2-\lambda) p_{N+1}(\lambda)-p_{N}(\lambda)
$$

with initial conditions $p_{0}(\lambda)=1$ (by definition), $p_{1}(\lambda)=2-\lambda$. However these equations are also satisfied by $U_{N}\left(1-\frac{1}{2} \lambda\right)$ so we must have

$$
P_{N}(\lambda)=U_{n}\left(1-\frac{1}{2} \lambda\right) \text {. }
$$

Now the zeros of $U_{N}(\cos \theta)=\sin (N+1) \theta / \sin \theta$ are at $\theta=n \pi /(N+1)(n-1, \ldots, N)$, therefore the zeros of $p_{N}(\lambda)=U_{N}\left(1-\frac{1}{2} \lambda\right)$ are at

$$
1-\frac{1}{2} \lambda=\cos \frac{n \pi}{N+1} \quad(n=1, \ldots, N)
$$

i.e.

$$
\begin{aligned}
\lambda & =2\left(1-\cos \frac{n \pi}{N+1}\right) \\
& =4 \sin ^{2} \frac{\frac{1}{2} n \pi}{N+1} \quad(n=1, \ldots, N) \\
& =4 \cos ^{2} \frac{\frac{1}{2} n \pi}{N+1} \quad(n=1, \ldots, N)
\end{aligned}
$$

(in decreasing order of magnitude).

Corollary. The eigen-values of the $N \times N$ matrix

are $\left\{4 \cos ^{2} \frac{\frac{1}{2} n \pi}{N+\frac{1}{2}}\right\}_{n=1,2, \ldots, N}$

$$
C_{N}=\left(\begin{array}{rrrrrr}
2 & -1 & & & & \\
-1 & 2 & -1 & & & \\
& -1 & 2 & \ddots & \\
& & \ddots & \ddots & -1 & \\
& & -1 & 2 & -1 \\
& & & & -1 &
\end{array}\right)
$$


Proof. Denoting the characteristic polynomial of $C_{N}$ by $q_{N}(\lambda)$ we have

$$
\begin{aligned}
q_{N}(\lambda) & =p_{N}(\lambda)-p_{N-1}(\lambda) \quad(N \geqslant 1) \\
& =U_{N}\left(1-\frac{1}{2} \lambda\right)-U_{N-1}\left(1-\frac{1}{2} \lambda\right) \\
& =(\sin (N+1) \theta-\sin N \theta) / \sin \theta
\end{aligned}
$$

(putting $1-\frac{1}{2} \lambda=\cos \theta$ )

$$
=\cos \left(N+\frac{1}{2}\right) \theta / \cos \frac{1}{2} \theta
$$

which vanishes at $\theta=\left(n-\frac{1}{2}\right) \pi / N+\frac{1}{2}(n=1,2, \ldots, N)$ and therefore at $1-\frac{1}{2} \lambda=$ $\cos \left(n-\frac{1}{2}\right) \pi /\left(N+\frac{1}{2}\right)(n=1,2, \ldots, N)$ i.e.

$$
\begin{array}{rlrl}
\lambda & =2\left(1-\cos \left(n-\frac{1}{2}\right) \pi / N+\frac{1}{2}\right) \\
& =4 \sin ^{2}\left(\frac{1}{2}\left(n-\frac{1}{2}\right) \pi / N+\frac{1}{2}\right. & (n=1, \ldots, N) \\
& =4 \cos ^{2}\left(\frac{1}{2} n \pi / N+\frac{1}{2} \quad\right. & (n=1, \ldots, N)
\end{array}
$$

in descending order of magnitude.

It is clear that the eigen-values of $A$ are the same as those of

$$
\left(\begin{array}{c|c}
C_{N} & 0 \\
\hline 0 & C_{N+1}
\end{array}\right)
$$

and so are $\left\{4 \cos ^{2}\left(\frac{1}{2} n \pi\right) / N+\frac{1}{2}\right\}_{n=1,2, \ldots, N}$ together with $\left\{4 \cos ^{2}\left(\frac{1}{2} n \pi\right) / N+\frac{3}{2}\right\}$ $n=1,2, \ldots, N+1$, which, on rearranging in descending order gives

$$
\begin{aligned}
& \nu_{n}=4 \cos ^{2} \frac{\left(\frac{1}{2} n+1\right) \pi}{2 N+3} \quad(n \text { even }), \\
& \nu_{n}=4 \cos ^{2} \frac{\frac{1}{2}(n+1) \pi}{2 N+1} \quad(n \text { odd }) .
\end{aligned}
$$

Therefore, taking $N=n+1$, we have

$$
\nu_{n} \geqslant 4 \cos ^{2} \frac{1}{4} \pi=2 .
$$

Hence

$$
\left|\lambda_{n}\right| \geqslant \mu_{n} \geqslant \frac{\pi}{8(n+1)} \nu_{n} \geqslant \frac{\pi}{4(n+1)}
$$

Combining this inequality with the one obtained in Section 3, we have

$$
\frac{1}{4} \pi \leqslant \underline{\lim } n\left|\lambda_{n}\right| \leqslant \overline{\lim } n\left|\lambda_{n}\right| \leqslant \pi
$$

which shows $\lambda_{n}$ to be of order $1 / n$ for large $n$, as claimed in Section 1 . 
6.

It remains to indicate how the above methods apply to the equation

$$
\int_{-1}^{1} \frac{1}{|x-t|^{\alpha}} f(t) d t=\lambda f(x) .
$$

Instead of Chebyshev polynomials, we use ultraspherical polynomials, defined by

$$
\frac{1}{\left(1-2 x t+t^{2}\right)^{\alpha}}=\sum_{n=0}^{\infty} P_{n}^{(\alpha)}(x) t^{n}
$$

The kernel has an expansion in terms of ultraspherical polynomials of order $\alpha / 2$, namely,

$$
\frac{1}{|x-t|^{\alpha}}=\frac{\Gamma((1-\alpha) / 2) \Gamma(1+\alpha / 2)}{\Gamma(1 / 2)} \sum_{n=0}^{\infty}(1+2 n / \alpha) P_{n}^{(\alpha / 2)}(x) P_{n}^{(\alpha / 2)}(t),
$$

which gives

$$
\int_{-1}^{1} \frac{1}{|x-t|^{\alpha}}\left(1-t^{2}\right)^{-(1-\alpha) / 2} P_{n}^{(\alpha / 2)}(t) d t=\frac{\Gamma((1+\alpha) / 2) \Gamma((1-\alpha) / 2)}{\Gamma(\alpha)} \frac{\Gamma(n+\alpha)}{\Gamma(n+1)} P_{n}^{(\alpha / 2)}(x)
$$

for all $n \geqslant 0$. (See (3) pages 27,28 ).

Proceeding as in Section 3, we find that the eigen-values of

$$
\int_{-1}^{1} \frac{1}{|x-t|^{\alpha}} f(t) d t=\lambda f(x)
$$

are all positive, and if we denote them by

$$
\lambda_{0} \geqslant \lambda_{1} \geqslant \lambda_{2} \geqslant \cdots
$$

then

for all $n$. Since

$$
\lambda_{n} \leqslant \frac{\Gamma((1+\alpha) / 2) \Gamma((1-\alpha) / 2)}{\Gamma(\alpha)} \frac{\Gamma(n+\alpha)}{\Gamma(n+1)}
$$

$$
\Gamma(n+\alpha) / \Gamma(n+1) \sim 1 / n^{1-\alpha},
$$

it follows that $\lambda_{n}=O\left(1 / n^{1-\alpha}\right)$. The methods of Sections 4 and 5 give $1 / n^{1-\alpha}=O\left(\lambda_{n}\right)$ by expanding $1 /|x-t|^{\alpha}$ in terms of ultraspherical polynomials of order $\alpha / 2+1$. Hence, as claimed in Section $1, \lambda_{n}$ is of order $1 / n^{1-\alpha}$ for large $n$.

\section{REFERENCES}

(1) M. KAC, Distribution of eigen-values of certain integral operators. Mich. Math. J. 3 (1955-6), 141-8.

(2) A. Pietsch, Nuclear Locally Convex Spaces (Springer-Verlag, 1972).

(3) G. PolyA, and G. Szego, Uber den transfiniten Durchmesser (Kapazitatskonstante) von ebenen und raumlichen Punktmengen, Crelle's Journal 165 (1931), $4-49$.

(4) M. RosenblatT, Some results on the asymptotic behaviour of eigenvalues for a class of integral equations with translation kernels, J. Math. Mech. 12 (1963), 619-628.

The UNIVERSITY OF MANChester 